\title{
Study of reticulated vitreous carbon foam as a quasi-brittle material
}

\author{
P. J. Heard ${ }^{1, a}$ and P. E. J. Flewitt ${ }^{1,2, b}$ \\ ${ }^{1}$ Interface Analysis Centre, School of Physics, University of Bristol, Bristol BS8 1TL, UK \\ ${ }^{2}$ School of Physics, HH Wills Physics Laboratory, University of Bristol, Bristol BS8 1TL, UK \\ aPeter.Heard@bristol.ac.uk ${ }^{b}$ Peter.Flewitt@bristol.ac.uk
}

Keywords: Carbon foam, Quasi-brittle, Tensile test, Stored energy, Fracture.

\begin{abstract}
Quasi-brittle materials are those where the addition of specific microstructural features such as porosity can lead to departure from linear elastic behaviour prior to maximum force, followed by graceful failure. A simple example of a quasi-brittle material is reticulated vitreous carbon foam; an open-cell structure consisting of brittle ligaments connected in a three-dimensional array. Tensile testing measurements have been made on foams with various pore and ligament dimensions; force - displacement combined with acoustic monitoring together with the evaluation of the associated elastic moduli and fracture strengths. These tests give insights into the mechanisms of quasi-brittle failure, and the results are explored using simple considerations of elastic energy storage throughout process zones.
\end{abstract}

\section{Introduction}

Quasi-brittle materials are those in which individual elements of the material exhibit a linear elastic behaviour but specific additional microstructural features, such as porosity, lead to departure from this behaviour. Hence larger components behave in an elastic fashion initially, but depart from linearity prior to peak load, followed by graceful failure. This class of materials includes cement, bone and the various graphite types used as moderators in nuclear power stations [1]. Under tensile or bending loads, "process zones" are said to form - regions in which local elastic and inelastic displacements are recorded prior to fracture. The inelastic component is usually a result of microcrack formation. As loading continues, the micro-cracks coalesce and eventually form macrocracks, and the component proceeds to failure. To fully understand quasi-brittle behaviour, it is necessary to link the small-scale micro-cracking behaviour and the formation of process zones, to the formation of macro-cracks, and hence a multi-scaled approach is required. For many of these materials the microstructure and composition on the small scale is very complex. Even for nuclear reactor core graphite such as Pile Grade A (PGA) or Gilsocarbon that contains carbon with minor impurities, the microstructure is complex over a range of length scales [2]. Here we consider a simpler material; that of reticulated vitreous carbon foam. The composition is simply carbon, but being vitreous there is little order on the atomic scale in contrast to graphite, and the individual ligaments would be expected to have linear elastic brittle fracture characteristics like glass. The ligaments are assembled in a three-dimensional array with four ligaments emanating from each node, to form an open-celled foam. Such materials are readily available with a range of pore and ligament sizes, and components up to tens of centimetres in size are available. Here, a set of reticulated vitreous carbon foam specimens have been subjected to tensile tests to establish if they behave as quasi-brittle materials, and whether simple energy considerations can be used to explain their behaviour.

\section{Experimental}

Samples of reticulated vitreous carbon foam were obtained from Ultramet [3], Fig. 1(a). Three pore sizes were chosen: 10 pore per inch (ppi), 45ppi and 100ppi. Samples were cut to produce cylindrical specimens of two sizes: $12 \mathrm{~mm}$ diameter x $12 \mathrm{~mm}$ long, and $25 \mathrm{~mm}$ diameter x $25 \mathrm{~mm}$ long. Cutting was either using a slow speed diamond wheel saw for flat surfaces, or a trepan tool for the cylindrical surfaces. Upon examination by scanning electron microscopy, the individual 

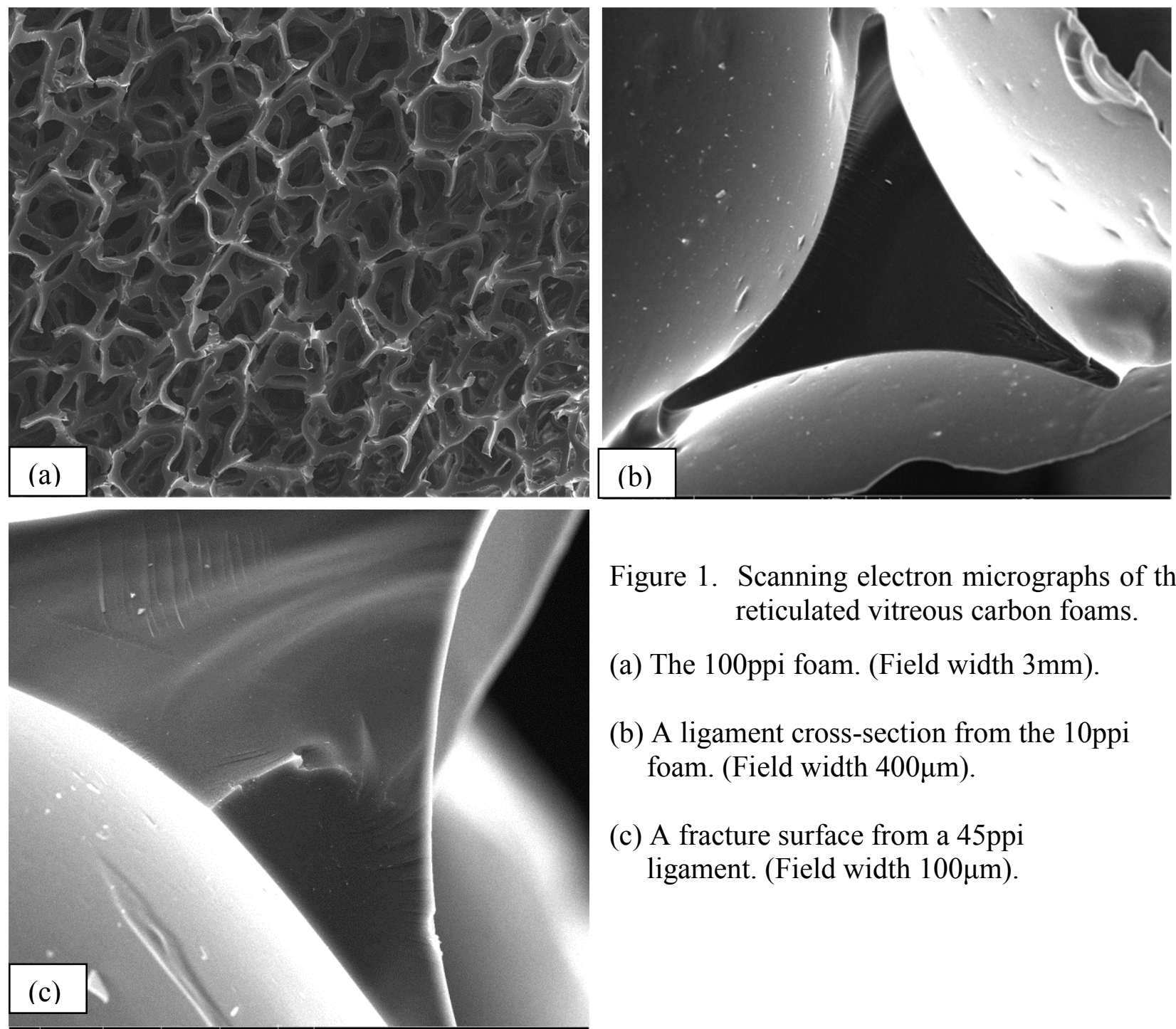

Figure 1. Scanning electron micrographs of the reticulated vitreous carbon foams.

(a) The 100ppi foam. (Field width 3mm).

(b) A ligament cross-section from the 10ppi foam. (Field width $400 \mu \mathrm{m}$ ).

(c) A fracture surface from a $45 \mathrm{ppi}$ ligament. (Field width $100 \mu \mathrm{m}$ ).

Table 1. Characteristics of reticulated vitreous carbon foams of 10, 45 and 100 pores per inch.

\begin{tabular}{|c|c|c|c|c|c|}
\hline Foam & $\begin{array}{c}\text { Measured } \\
\text { pore size } \\
{[\mathbf{m m}]}\end{array}$ & $\begin{array}{c}\text { Ligament } \\
\text { tip to tip } \\
\text { distance }[\boldsymbol{\mu m}]\end{array}$ & $\begin{array}{c}\text { Ligament } \\
\mathbf{x} \text {-sectional area } \\
{\left[\boldsymbol{\mu m}^{\mathbf{2}}\right]}\end{array}$ & $\begin{array}{c}\text { Ligament } \\
\text { length } \\
{[\mathbf{m m}]}\end{array}$ & $\begin{array}{c}\text { Average foam } \\
\mathbf{d e n s i t y} \\
{\left[\mathbf{g ~ c m}^{-3}\right]}\end{array}$ \\
\hline $\mathbf{1 0 p p i}$ & 2.5 & 300 & 24000 & 2.1 & 0.047 \\
\hline $\mathbf{4 5 p p i}$ & 1.0 & 160 & 6300 & 0.7 & 0.054 \\
\hline $\mathbf{1 0 0 p p i}$ & 0.45 & 58 & 900 & 0.2 & 0.057 \\
\hline
\end{tabular}

ligaments are approximately triangular in cross-section as show in Figure 1(b). Table 1 shows the measured physical properties of each of the materials used. The ends of the cylindrical specimens were glued to aluminium plates using a stiff, fast-drying adhesive ( 5 minute epoxy). One plate was glued to a cylinder end initially and the assembly was mounted in the tensile tester. A second plate was then glued to the other end of the cylinder while the specimen was in the tester so as to minimise mounting strain on the samples. After the glue had dried for at least an hour, tensile testing commenced. A Zwick/Roell tensile tester was used in these experiments. Force and displacement were measured during the tests, which were displacement controlled. A displacement rate of $0.1 \mathrm{~mm} /$ minute was used throughout. An Olympus LS-10 sound recorder was placed 
approximately $2 \mathrm{~cm}$ from the specimen during testing in order to record any acoustic emissions. A simple USB camera was used to record images of the specimen at 1s intervals during testing.

\section{Results}

A scanning electron micrograph of the fracture surface of individual ligaments for the 10ppi and 45ppi foams are shown in Figures 1(b) and 1(c). The fracture surfaces are similar to that of glass, displaying striations and smooth regions consistent with brittle fracture. Typical force-displacement curves recorded for the $12 \mathrm{~mm}$ specimen size at 10ppi and 100ppi pore sizes are shown in Figure 2. In addition, acoustic emission traces are shown beneath each trace. The specimen of 10 pores per inch, Figure 2(a), displays discrete fracture events that are also recorded acoustically. Peak force is approximately $30 \mathrm{~N}$, reached at a displacement of $0.11 \mathrm{~mm}$. Post-peak force, further fracture events are recorded and force approximates to zero at a displacement of $0.3 \mathrm{~mm}$. The 100ppi specimen, Figure 2(b) however, displays almost linear behaviour until fast fracture occurs at a force of $45 \mathrm{~N}$ and a displacement of $0.25 \mathrm{~mm}$. No further events are recorded after failure, and the force drops to zero.

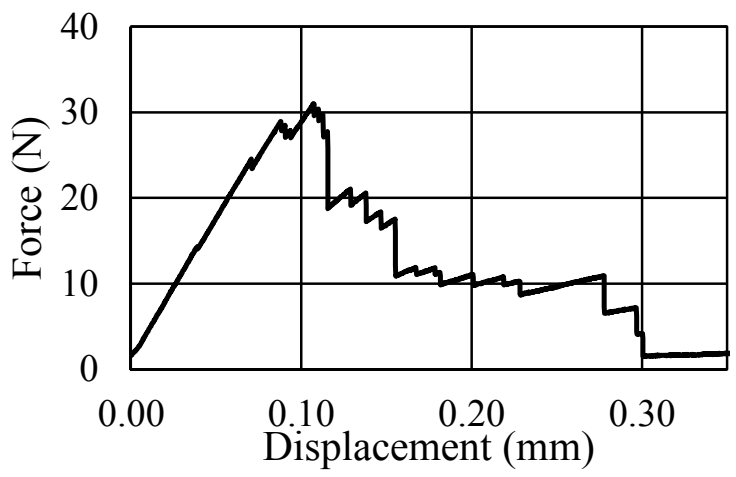

a)

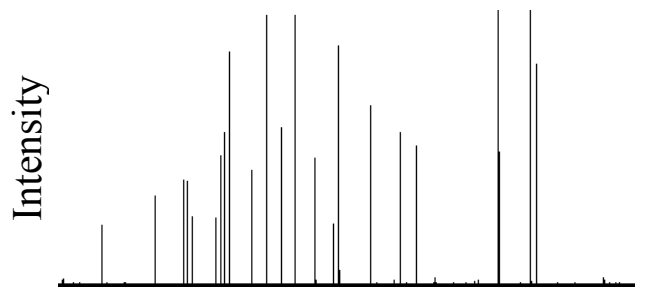

Displacement (mm)

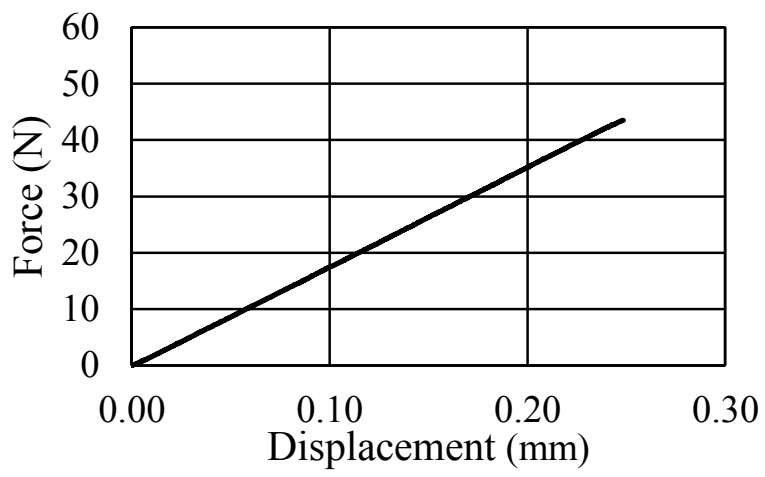

b)

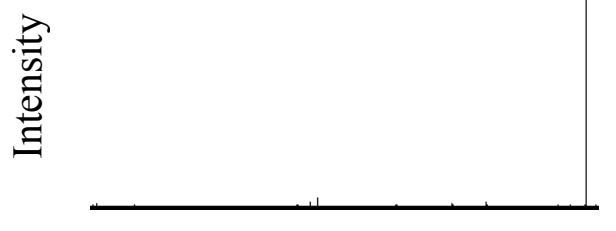

Displacement (mm)

Figure 2. Force-displacement curves with associated acoustic emission traces for two specimens:
a) 10 ppi $12 \mathrm{~mm}$
b) 100 ppi $12 \mathrm{~mm}$

\section{Concluding comments}

Table 2 shows the stored elastic energy density in the material at peak force, with the energy stored per pore, the elastic modulus and the tensile strength. For the coarse (10ppi) foam specimens, and to a lesser extent the 45ppi foams, the force changes during the tensile test can be measured, and these correspond to breakages of the ligaments in the specimen. As ligaments break and tensile testing continues, the force-displacement traces remain linear such that the trace segments can be extended back to the graph origin. This indicates that the specimen is behaving simply as a spring, and as each ligament breaks, the spring constant is reduced. By measuring the force changes and the corresponding displacements, the energy released by the fracture of each ligament can be calculated by:

$$
\mathrm{Q}=1 / 2 \Delta \mathrm{F} \cdot \mathrm{X}
$$

where $\Delta \mathrm{F}$ is the change in force and $\mathrm{x}$ is the displacement at that point. 
As each ligament breaks, a small amount of released energy will have been used in separating the two faces of the fractured ligament, breaking chemical bonds to do so. The majority of the energy will be dissipated as heat and sound, as the surfaces separate rapidly, oscillate for a short while and come to rest. Optical images obtained during the tensile test show the specimen to "reconfigure" elastically after each fracture event, so that ligaments in the vicinity of the fracture move slightly, the forces within the matrix are once more balanced and mechanical equilibrium is reached. For the $12 \mathrm{~mm} \mathrm{10ppi} \mathrm{specimen,} \mathrm{the} \mathrm{fracture} \mathrm{release} \mathrm{energies} \mathrm{range} \mathrm{from} 0.04 \mathrm{~mJ}$ to $0.6 \mathrm{~mJ}$. As each fracture event occurs, a small region of the surrounding material moves to accommodate the energy release, so that this region can be regarded as a "process zone". As process zones overlap and fractures occur close to each other, it is more likely that failure of the specimen will occur. The 100ppi specimens display rather different behaviour, despite being composed of the similar vitreous material. The difference between this foam and the others is the distribution of the ligaments, pore volume/size, Tables 1 and 2. Here, the force-displacement curve is much more linear to the peak maximum, and failure is prompt.

Table 2. Maximum stored energy per unit volume and per pore for each specimen size and pore size, elastic modulus and tensile strength.

\begin{tabular}{|c|c|c|c|c|c|c|}
\hline $\begin{array}{c}\text { Pores } \\
\text { per inch }\end{array}$ & $\begin{array}{c}\text { Specimen } \\
\text { size } \\
{[\mathbf{m m}]}\end{array}$ & $\begin{array}{c}\text { Max Stored } \\
\text { Energy per unit } \\
\text { volume }\left[\mathbf{J} / \mathbf{m}^{\mathbf{3}}\right]\end{array}$ & $\begin{array}{c}\text { Pore } \\
\text { Volume } \\
{\left[\mathbf{m m}^{\mathbf{3}}\right]}\end{array}$ & $\begin{array}{c}\text { Max Stored } \\
\text { Energy per } \\
\text { pore }[\mathbf{m J}]\end{array}$ & $\begin{array}{c}\text { Elastic } \\
\text { Modulus } \\
{[\mathbf{M P a}]}\end{array}$ & $\begin{array}{c}\text { Tensile } \\
\text { Strength } \\
{[\mathbf{M P a}]}\end{array}$ \\
\hline 10 & 12 & 1358 & 15.6 & 0.021 & 32.3 & 0.26 \\
\hline 10 & 25 & 1983 & 15.6 & 0.030 & 34.2 & 0.30 \\
\hline 45 & 12 & 2379 & 1.0 & 0.0024 & 50.2 & 0.43 \\
\hline 45 & 25 & 3318 & 1.0 & 0.0034 & 31.7 & 0.41 \\
\hline 100 & 12 & 5344 & 0.091 & 0.00048 & 15.6 & 0.40 \\
\hline 100 & 25 & 5793 & 0.091 & 0.00053 & 11.9 & 0.36 \\
\hline
\end{tabular}

The use of such simple structures for mechanical testing of this type lends itself to computer modelling, and future work will include a comparison of such models with the experimental results obtained here. It is hoped that a study of these simple materials will shed light on the behaviour of more complex materials such as graphite, bone and cement, and elucidate the failure mechanisms of quasi-brittle materials as a whole.

Acknowledgements. The authors acknowledge the financial support from the EPSRC grants EP/J019801/1 (Bristol): QUBE: QUasi-Brittle fracture: a 3D Experimentally-validated approach.

\section{References}

[1] A. Hodgkins, T.J. Marrow, M.R. Wootton, R. Moskovic and P.E.J. Flewitt, Mat. Sci. Tech. 26 (2010) 889-907.

[2] P.J. Heard, M.R. Wootton, R. Moskovic, P.E.J. Flewitt, J. Nucl. Mater, 401 (2010) 71-77.

[3] Ultramet, Pacoima, CA., USA 